\title{
Detecting the Presence of Fog Using Low-cost Proximity Sensors
}

\author{
Derek J. Straub* \\ Department of Earth and Environmental Sciences, Susquehanna University, Selinsgrove, PA 17870, USA
}

\begin{abstract}
A new, low-cost device that detects the presence of fog has been developed for applications such as the activation of fog sampling equipment and the monitoring of fog's spatial or temporal evolution. This fog presence detector (FPD) offers an inexpensive alternative to visibility monitors and other specialized fog or cloud microphysical instruments that have typically been used for these purposes. The FPD utilizes a VCNL4200 proximity sensor (Vishay Intertechnology, Inc.), which operates as an infrared backscatter detector and was intended to detect proximity in cars, buildings, and personal electronics. An FPD with six individual sensors was evaluated in the field from 19 August 2019 through 4 November 2019. Over the course of the study, the FPD was able to distinguish between foggy and clear conditions. The output values from the individual sensors closely tracked one another and were strongly correlated with extinction coefficient measurements from a collocated visibility monitor. However, the study revealed that a more powerful, external infrared emitting diode (IRED) was necessary for reliable fog detection when sunlight was present. With the inclusion of an external IRED, the agreement between the FPD and visibility monitor averaged $91 \%$ when indicating the presence of fog during fog events. Both the FPD and visibility monitor were found to be susceptible to insects and spider webs located in or near the optical sensing volume during foggy and non-foggy periods.
\end{abstract}

Keywords: Low-cost sensors; Radiation fog; Visibility; Liquid water content; Infrared backscatter.

\section{INTRODUCTION}

The ability to detect the presence of fog can be a critical requirement for field-based studies of fog chemical composition, fog climatology, or fog dynamics. Sensors that indicate the time of fog formation and dissipation have been used to automate systems for the collection of fog water or to document spatial and temporal variations in fog occurrence. In situ fog detection has typically been performed with visibility monitors or specialized cloud microphysical instruments. While effective, these instruments can be expensive and cost-prohibitive for large or dense fog monitoring networks or for low-cost applications. This study investigates a low-cost approach for fog detection based on an inexpensive infrared (IR) proximity sensor originally designed for personal electronic devices and presence detection in buildings. In addition to low cost, advantages of the new design include small size, portability, and low power consumption.

Fog consists of liquid water droplets suspended in air near the earth's surface. Droplets form when water vapor condenses onto particles that serve as condensation nuclei. This can

\footnotetext{
${ }^{*}$ Corresponding author.

E-mail address: straubd@susqu.edu
}

happen in response to a decrease in air temperature and associated decrease in saturation vapor pressure or it can occur as a result of moisture addition and associated increase in vapor pressure. Either mechanism can lead to saturation and droplet formation. Fog droplet sizes generally range between several micrometers and several tens of micrometers in diameter, differentiating them from smaller unactivated haze particles and larger precipitation-sized drops. Because fog droplets scatter light effectively, optical methods have generally been used for fog detection.

Fog is often defined in terms of visibility reduction, with operational thresholds of $1 \mathrm{~km}$ or 5/8 mile (NOAA, 2017; WMO, 2018), making visibility monitors a logical choice for detecting the presence of fog. Most visibility monitors are designed to measure scattering in the forward direction, commonly at angles of $42^{\circ}$ or $45^{\circ}$. They use an IR transmitter and receiver that are offset from one another to create a sensing volume where the two fields of view overlap. When fog droplets are present in the sensing volume, light is scattered toward the receiver which generates a signal that is converted to a visual range. Visibility monitors have been used to activate fog sampling equipment based on visibility thresholds ranging from $300 \mathrm{~m}$ to $1000 \mathrm{~m}$ (Thalmann et al., 2002; Burkard et al., 2003; Michna et al., 2013; Michna et al., 2015; Simon et al., 2016; Straub, 2017; Tav et al., 2018). Visibility monitors have also been used for studies of fog climatology and fog classification (Meyer and Lala, 
1990; Friedlein, 2004; Forthun et al., 2006; Herckes et al., 2007; Tardif et al., 2007; Van Schalkwyk and Dyson, 2013; van Oldenborgh et al., 2010; Giulianelli et al., 2014).

The Gerber PVM-100 measures fog or cloud liquid water content (LWC) and has also been used for detecting fog presence during field-based fog studies (Baumgardner et al., 1997; Facchini et al., 1999; Gelencsér et al., 2000; Ervens et al., 2013; Giulianelli et al., 2014; Boris et al., 2016; Decesari et al., 2017). The PVM-100 employs a $780 \mathrm{~nm}$ laser diode to illuminate a population of droplets over a $42 \mathrm{~cm}$ path length and measures scattering in the near-forward direction. A precisely designed variable transmission filter ensures that the scattered light is proportional to LWC. Because the PVM-100 reports LWC, and is one of the few commercially available instruments to do so, it provides valuable information beyond just a fog/no-fog determination and therefore justifies its high cost if the study objectives require such information.

The Colorado State University Optical Fog Detector (CSU-OFD) was developed as a low-cost LWC monitor (Carrillo et al., 2008) and has also been used to trigger fog collection systems in the presence of fog (Carrillo et al., 2008; Straub et al., 2012; Ervens et al., 2013; Wang et al., 2015). The CSU-OFD relies on the attenuation of $880 \mathrm{~nm}$ light along a $53.3 \mathrm{~cm}$ path length between an infrared emitting diode (IRED) transmitter and a photodiode receiver. For the components used in the CSU-OFD, light attenuation rather than scattering was found to produce a signal that was correlated with PVM-100 LWC measurements. Although some interference from rain and an oversensitivity to small drops was noted, the CSU-OFD can indicate the presence of fog as well as provide estimates of LWC. After operating the CSU-OFD continuously over extended sampling periods, Straub et al. (2012) confirmed the CSU-OFD's sensitivity to rainfall and also noted issues related to baseline signal drift that impacted fog detection. The CSU-OFD is not currently in production and is not commercially available.

Finally, an innovative low-cost fog detection system pairs a $1 \mathrm{~m}^{2}$ standard mesh passive fog collector and an optical rain sensor (Weiss-Penzias et al., 2018). Fog detection occurs when fog droplets that are transported by the wind impact the mesh collector, drain through a trough, and are directed to an optical rain sensor. As the first drops of water roll down the surface of the rain sensor, IR light scattering internal to the sensor is disrupted, indicating the presence of liquid water. While effective for coastal or other types of fog in which droplets are transported by the wind, the design may not be suitable for radiation fog where calm conditions would prevent the transport, impaction, and accumulation of fog droplets on the mesh strands.

With the proliferation of miniaturized sensors for automation and smart devices, there are numerous opticalbased proximity sensors on the market. Because of similar functionality to some of the sensors described above, the potential exists for a proximity sensor to be repurposed as a miniaturized, low-power, very low-cost fog detector. With this in mind, several IR proximity sensors were evaluated for their ability to reliably differentiate between clear and foggy conditions. Two sensors, the MAX30105 (Maxim Integrated) and VCNL4020 (Vishay Intertechnology, Inc.) suffered from high background noise, a limited response in the presence of fog, or substantial signal drift over time, making them unsuitable for fog detection. A third sensor, the VCNL4200 (Vishay Intertechnology, Inc.), showed the greatest potential for use as a fog detector, with an in-fog response that was distinguishable from clear air and an amplitude that was well correlated with visibility measurements. Field tests indicated that the sensor was durable and performed well over time, although a sensitivity to insects and spider webs in the optical sensing volume was also noted. The development and evaluation of the new VCNL4200-based fog presence detector (FPD) will be described in the following sections.

\section{METHODS}

\section{Instrument Design}

The new FPD relies on the VCNL4200 long-range proximity sensor (Vishay, 2019), which consists of a $940 \mathrm{~nm}$ infrared emitting diode and a photodiode receiver housed with associated electronics in a small integrated circuit (IC) package. The VCNL4200 has an $8 \mathrm{~mm} \times 3 \mathrm{~mm}$ footprint and a height of $1.8 \mathrm{~mm}$ (Fig. 1(a)). The IRED and receiver are both mounted on the top of the package and proximity measurements are made by emitting pulses of IR light at a target object and measuring the strength of the backscattered signal. The use of backscattered light for fog detection eliminates the need for separate emitter and receiver components that must be spaced some distance apart, as is the case for instruments that rely on forward scattering or attenuation. That, combined with the very small footprint of the VCNL4200, allows for a much more compact and portable design. The angle of half intensity of the IRED is $15^{\circ}$, and the receiver has a $\pm 30^{\circ}$ field of view. A standard $\mathrm{I}^{2} \mathrm{C}$ interface provides communications with the VCNL4200.

The VCNL4200 sensor was mounted on one side of a $3.4 \mathrm{~cm}$ square printed circuit board so that it could be exposed to ambient conditions while the supporting electronics were placed on the opposite side (Fig. 1(b)). An acrylic conformal coating (419D; MG Chemicals) was applied to both sides of the board to prevent water infiltration and corrosion. The technical specifications of the FPD are summarized in Table 1.

Two sensor configurations were evaluated. One configuration relied on the VCNL4200's internal IRED which could be driven with a maximum current of $800 \mathrm{~mA}$. A second configuration bypassed the internal IRED and instead utilized a more powerful external $940 \mathrm{~nm}$ IRED (VSMY99445DS; Vishay Intertechnology, Inc.) that could be driven with a higher current (Fig. 1(c)). A drive current of 2 A was used for this configuration in an effort to increase illumination and therefore enhance the backscatter signal in fog. Compared to the VCNL4200's internal IRED, the external IRED had a larger angle of half intensity of $45^{\circ}$ which increased the volume of air that was sensed.

The VCNL4200 includes a background light cancellation feature that can be used to reduce or eliminate interference from background IR sources. Initial testing showed that even low levels of indirect sunlight caused the sensor signal to become saturated. Therefore, the background light 

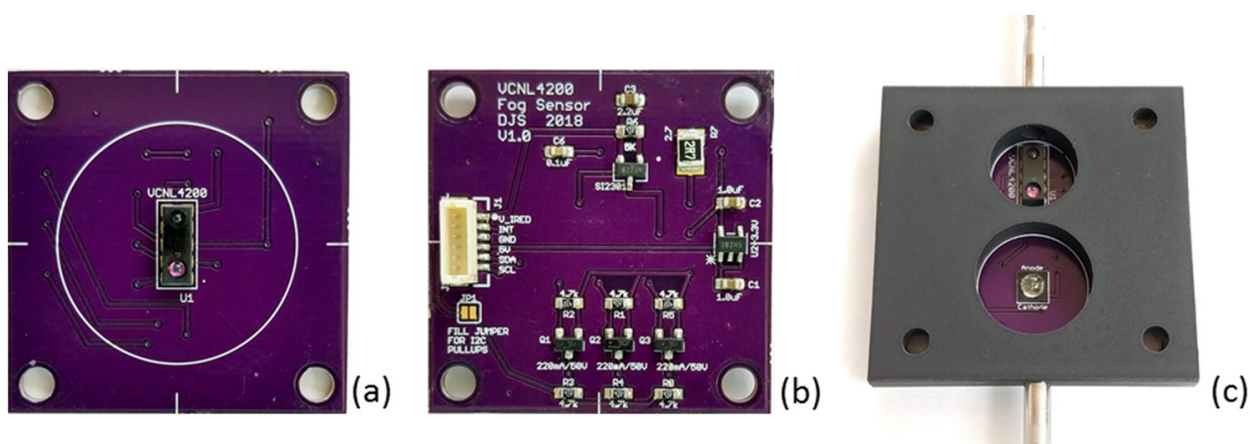

Fig. 1. (a) VCNL4200 sensor mounted on the front of a PCB for use as a fog presence detector (FPD). (b) The sensor is $8 \mathrm{~mm} \times 3 \mathrm{~mm}$, and the PCB is $3.4 \mathrm{~cm} \times 3.4 \mathrm{~cm}$. Supporting electronics were placed on the back of the PCB. (c) An FPD sensor with external IRED mounted behind a $4.8 \mathrm{~mm}$ thick aluminum plate with tubing that directs air at $1 \mathrm{~L} \mathrm{~min}^{-1}$ over the optical components.

Table 1. FPD technical specifications.

\begin{tabular}{ll}
\hline Parameter & Value \\
\hline VCNL4200 dimensions & $8 \mathrm{~mm} \times 3 \mathrm{~mm} \times 1.8 \mathrm{~mm}$ \\
Emitter wavelength & $940 \mathrm{~nm}$ \\
Detector field of view & $\pm 30^{\circ}$ \\
Emitter angle of half intensity & $\pm 15^{\circ}$ (int. IRED)/ $\pm 45^{\circ}$ (ext. IRED) \\
Measurement frequency & $1 \mathrm{~Hz}$ \\
Output & $16 \mathrm{bit}$ ADC $(0-65,535$ counts) \\
Communications & $\mathrm{I}^{2} \mathrm{C}$ \\
Power consumption (VCNL4200/supporting electronics/pump) & $0.02 \mathrm{~W} / 0.5 \mathrm{~W} / 1.2 \mathrm{~W}$ \\
Recommended threshold for fog detection (internal/external IRED) & 5 counts/10 counts \\
\hline
\end{tabular}

cancellation feature was enabled for all evaluations of the FPD so that measurements could be made during the daytime. This capability is critical as fog in this region routinely persists after sunrise and occasionally forms after sunrise. For the FPD sensors that used the VCNL4200's internal IRED, however, the background light cancellation feature appears to have been sufficiently aggressive so that when sunlight was present, the desirable backscatter signal from fog was reduced or eliminated along with the elimination of the background solar input. Fog could be detected using the internal IRED configuration before sunrise, but if the fog persisted after sunrise, the signal was reduced or even lost. This was not an issue for the FPD configured with the external IRED.

A microcontroller was required to initialize the VCNL4200 sensor, to request measurements, and for data acquisition and processing. In keeping with a low-cost approach, an Arduino-compatible microcontroller (Metro Mini; Adafruit Industries) was used for this purpose. For data storage, a microSD card writer was employed along with a precision real-time clock for time stamping. Measurements were made at $1 \mathrm{~Hz}$ throughout the study. To avoid interference between adjacent sensors, the sensors were triggered sequentially with a $30 \mathrm{~ms}$ delay. At this sample rate, the average power consumption for each FPD sensor was $0.022 \mathrm{~W}$. The measured power consumption for the data acquisition and control electronics was approximately $0.5 \mathrm{~W}$.

The individual components of the FPD were all readily available and inexpensive to purchase. The VCNL4200 sensors and external IREDs cost less than 6 USD each while the remaining components of the FPD were purchased for under 150 USD. This represents a low-cost alternative to commercially available visibility monitors with prices in the thousands of dollars or specialized microphysical instruments with prices in the tens of thousands of dollars.

\section{Experimental Setup}

The evaluation of the FPD occurred between 19 August and 4 November 2019 at Susquehanna University's Center for Environmental Education and Research (CEER) located in central Pennsylvania. This site is situated in a broad river valley at an elevation of $160 \mathrm{~m}$ AMSL and predominantly experiences radiation fog which forms when light winds and clear skies promote strong radiational cooling at night (Roach et al., 1976; Gultepe, 2007). Fog also occasionally develops after sunrise at the test site and may be classified as morning evaporation fog (Tardif and Rasmussen, 2007), characterized by simultaneous increases in surface temperature and dew point after sunrise. Conditions conducive to both of these fog types are most common from mid-August through the end of October in this region. During the investigation period, 19 fog episodes occurred, lasting between 20 minutes and 7.5 hours each. These fog events were used as the basis for evaluating the performance of the FPD.

Six FPD sensors, spaced $10 \mathrm{~cm}$ apart in a single enclosure, were placed in the field for evaluation. Three sensors were configured with internal IREDs and three with external 
IREDs. Each fog sensor PCB was mounted behind a $4.8 \mathrm{~mm}$ thick aluminum plate with circular openings through which the optical components could view the environment (Fig. 1(c)). This recessed arrangement helped shield the optics from direct sunlight and also provided space for tubing that directed a flow of air at approximately $1 \mathrm{~L} \mathrm{~min}^{-1}$ over the optical components to prevent condensation and droplet deposition on the lenses. The power required by the miniature diaphragm pumps varied with the desired flow rate, but operation at $2.2 \mathrm{~L} \mathrm{~min}^{-1}$ required $1.2 \mathrm{~W}$.

The enclosure containing the test sensors was mounted $1 \mathrm{~m}$ above the ground. Preliminary observations indicated that the FPD sensors were sensitive to rain, most likely from water dripping off the enclosure in front of the sensor's optics, so a simple plywood cover extending $40 \mathrm{~cm}$ in front of the sensors was constructed. After installation there was no interference from rain over the duration of the study.

The FPD was collocated with a Belfort Model 6500 visibility monitor (Belfort Instrument Company) that relied on forward scattering of $890 \mathrm{~nm}$ wavelength light at an angle of $42^{\circ}$. The Belfort Model 6500 has a stated visibility measurement range between $6 \mathrm{~m}$ and $80 \mathrm{~km}$ and a stated accuracy of $\pm 10 \%$. This visibility monitor has been used at the test site for active control of an automated fog water collection system for the past 9 years. A visibility threshold of $500 \mathrm{~m}$ has been a reliable indicator of sufficiently dense fog for sample collection while excluding heavy rain events that can reduce visibilities to values between $500 \mathrm{~m}$ and $1000 \mathrm{~m}$ (Straub et al., 2012). For the purposes of the current study, the visibility monitor served as a benchmark for FPD evaluation.

Also located at the test site was a temperature and relative humidity sensor (HMP60; Vaisala Inc.), a tipping buck rain gauge (TE525WS-L; Texas Electronics Inc.), and an automated Caltech Heated Rod Cloudwater Collector (CHRCC). The CHRCC obtained fog samples through aerodynamic impaction of fog droplets on $3.2 \mathrm{~mm}$ diameter rods (Demoz et al., 1996). The CHRCC operated at an air flow rate of $5.8 \mathrm{~m}^{3} \mathrm{~min}^{-1}$ and had a theoretical $50 \%$ cut-off diameter of $9 \mu \mathrm{m}$. One fog sample was obtained per fog event and the collected mass was used to investigate the relationship between fog LWC and the response of the FPD.

\section{RESULTS AND DISCUSSION}

For the operation of the VCNL4200 proximity sensor as an FPD, fog droplets must scatter sufficient light back to the photodiode so that the output signal is indicative of the presence of fog. The signal in this case was the digital output from the VCNL4200's integral 16-bit analog to digital converter. The digital output range was between 0 counts when no backscattered light was detected and $2^{16}$ counts when the sensor was saturated. Electrical noise, the background light cancellation function, and reflection of IR light from surfaces surrounding the VCNL4200's optics all contributed to a background output signal that varied slightly over the course of a day. The internal IRED configuration produced background readings in the range of $0-3$ counts while the external IRED configuration, with its more powerful illumination and wider angle of half intensity, produced a background output that varied by about \pm 15 counts with an average magnitude of $60-80$ counts that varied by sensor. To put all the sensor measurements on a common basis, a smoothed background signal was subtracted from the total signal. Smoothing was accomplished with an exponentially weighted moving average that could be used for real-time baseline correction:

$S_{t}=\left(\alpha \cdot x_{t}\right)+(\alpha-1) \cdot S_{t-1}$

The smoothed background signal at time $t, S_{t}$, was a weighted average of the observed backscatter signal at time $t, x_{t}$, and the previous smoothed background signal, $S_{t-1}$. A weighting factor, $\alpha$, of 0.05 (internal IRED sensors) and 0.02 (external IRED sensors), produced a baseline that represented the slowly varying background signal.

\section{Performance in Foggy Conditions}

Based on the signal from the VCNL4200, it was possible to clearly distinguish between foggy and clear air conditions. Fig. 2 shows an example of the response of the six FPD sensors during a fog episode that occurred on 19 September 2019. Fog formed at approximately 6:00 AM as indicated by the increase in output signal above background levels across all six sensors. Fog density increased rapidly at 7:30 AM and dissipated at about 9:15 AM. The three sensors using external IREDs exhibited a greater response than the three sensors with internal IREDs. Very good agreement was observed among both sets of sensors, suggesting that the FPD is capable of responding to fog, including rapid fluctuations, in a repeatable way.

While good agreement between FPD sensors was typical, there were occasions when output from one or more of the

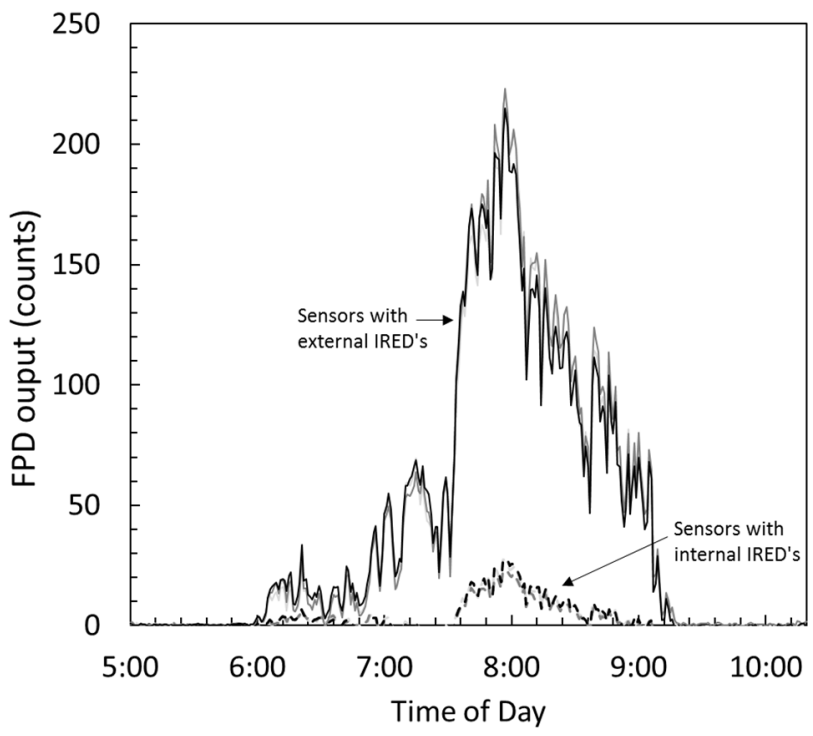

Fig. 2. The response of the FPD to a fog event on 19 September 2019. 1-minute-averaged output from the VCNL4200's analog-to-digital converter (ADC) is shown for three sensors with internal IREDs (dashed lines) and three sensors with external IREDs (solid lines). 
sensors differed significantly from the others. This study was conducted entirely in the field and therefore was susceptible to interference by insects and spider webs. When either was located in front of the optical components, the sensor's output signal was elevated. This occurred occasionally in foggy and non-foggy conditions. Shorter duration, higherintensity anomalous signals were attributed to insects passing across the sensor, while longer duration, lower-intensity signals occurred when spider webs were observed. When the optics were unobstructed and fog was present, however, the individual sensors of the FPD were strongly correlated with one another. Based on 1-minute-averaged data, statistically significant $(\mathrm{p}<0.01)$ correlation coefficients between each pair of sensors were greater than 0.97 for those with internal IREDs and greater than 0.99 for those with external IREDs. Linear regressions between pairs of FPD sensors resulted in slopes between 0.97 and 1.05 (internal IREDs) and between 0.97 and 1.03 (external IREDs).

To place the fog sensor data in context, comparisons were made to observations from the collocated visibility monitor. Although fog is often defined in terms of visibility, and thresholds for fog presence detection are typically given in terms of visibility, the Belfort Model 6500's optical output is linearly proportional to the extinction coefficient $\left(b_{\text {ext }}\right)$. Visibility is then calculated from $b_{\text {ext }}$ according to the Koschmieder equation:

$$
C=e^{\left(-b_{e x t} \cdot x\right)}
$$

where $C$ is the visual contrast ratio and $x$ is visibility. The Belfort Model 6500 assumes a contrast ratio of 0.05 ; therefore, the relationship between $b_{\text {ext }}$ and visibility can be approximated as follows:

$x=\frac{3.0}{b_{e x t}}$

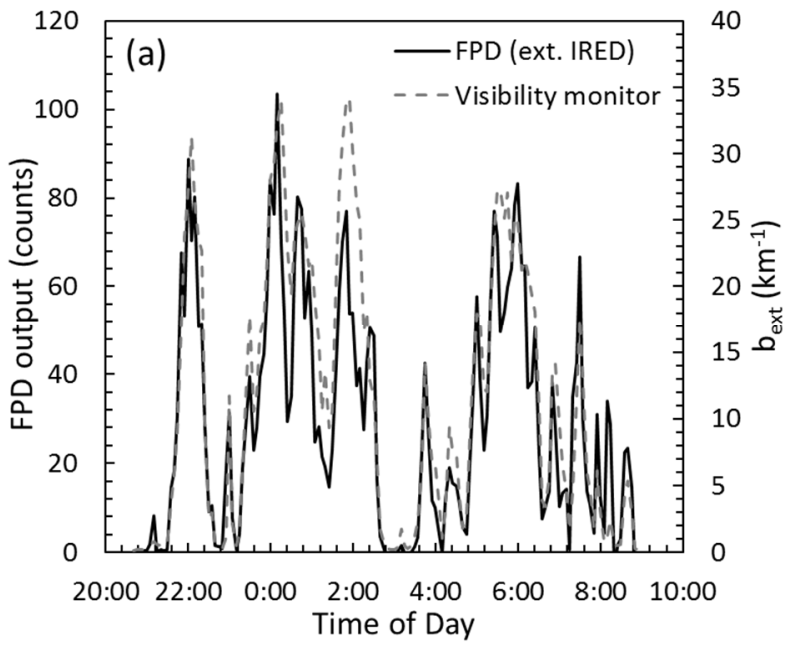

Fig. 3. 5-minute-averaged FPD (solid lines) and visibility monitor (dashed lines) data for two fog events. (a) Good agreement was observed on 23 October 2019 for a sensor with an external IRED. (b) A sensor with an internal IRED shows good agreement with the visibility monitor before sunrise on 21 September 2019, but the signal is diminished after sunrise.

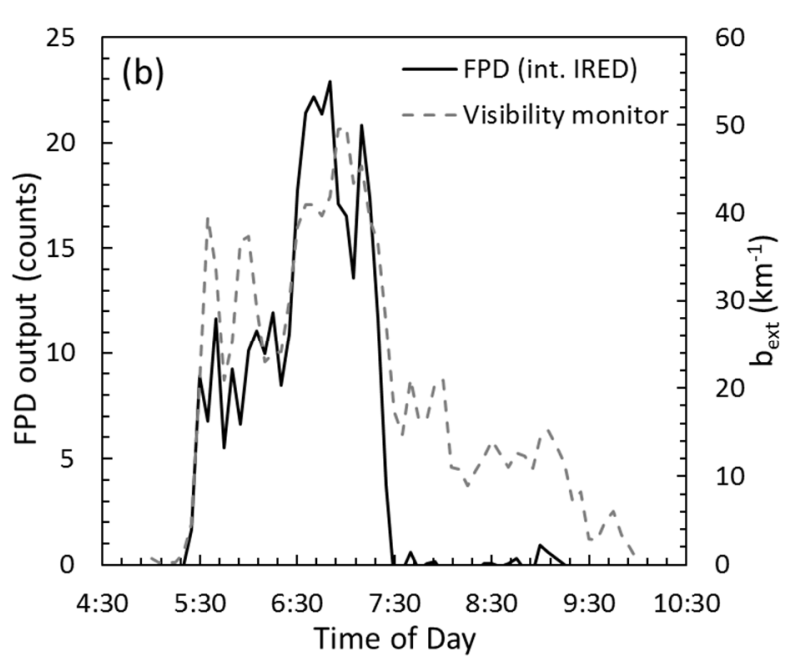

The extinction coefficient was used for comparison to the FPD sensor output because both increase in the presence of fog, i.e., as visibility decreases. Data from the visibility monitor was obtained at $0.5 \mathrm{~Hz}$ and recorded as 5-minuteaveraged values. For direct comparison, 5 minute averages were also calculated for the FPD data. Fig. 3 presents $b_{\text {ext }}$ values from the visibility monitor and baseline-corrected output from the FPD over the duration of two fog events. Fig. 3(a) shows data from a long duration fog event that occurred on 23 October 2019, and indicates that the external IRED version of the FPD is capable of closely tracking $b_{\text {ext }}$ throughout the duration of the fog event. Fig. 3(b) shows data for the internal IRED version of the FPD for a fog event that occurred on 21 September 2019. The internal IRED sensor tracked $b_{\text {ext }}$ during the first half of the event. After sunrise, however, the FPD output falls to zero and no longer responds to the fog. Similar behavior was observed whenever fog was present after sunrise, resulting in a loss of signal when fog was optically thin and a reduced signal when fog was thicker. As stated earlier, this appears to be the result of the background light cancellation function which significantly impacted the sensors that relied on the internal IREDs but not those coupled with an external IRED.

Similar to comparisons among the individual FPD sensors, there were times when FPD output and $b_{\text {ext }}$ differed significantly as a result of insects or spider webs on or near the optical components of either device. Because of the layout of the visibility monitor and its smaller sensing volume, it was less affected by these interferences. In clear air, the presence of spider webs reduced apparent visibility, but rarely to the threshold that would indicate fog. When spider webs were present on or near the visibility monitor optics in foggy conditions, however, observations were made of fog droplets adhering to the webs and persisting there even after fog dissipation. This resulted in unrealistically high values of $b_{\text {ext }}$ during the fog event or an overestimation of fog duration. These effects were observed during four of the 19 fog events. 
Fig. 4 shows correlations between $b_{\text {ext }}$ and output from one FPD sensor configured with an internal IRED and one sensor configured with an external IRED. The data presented in Fig. 4 were obtained during foggy periods, excluding the four events in which the visibility monitor returned anomalous readings as a result of observed spider webs. The data for the internal IRED sensor excludes one additional fog event in which observed spider webs impacted its measurements. The external IRED sensor was unobscured during all of the fog events. The FPD output and $b_{\text {ext }}$ exhibited strong, statistically significant correlations $(\mathrm{p}<$ 0.01 ), with $r=0.83$ and $r=0.93$ for the internal IRED and external IRED configurations, respectively. The lower correlation coefficient for the internal IRED was related primarily to the overcorrection of the backscatter signal by the background light cancellation function after sunrise. The scatter in Fig. 4 may be due to actual differences in fog density present at the instruments' respective locations but may also be attributed to the different scattering regimes employed by the instruments, potential multiple scattering effects in the larger sensing volume of the FPD, and differences in the quality and type of optical components used in the two instruments.

\section{Determination of fog Formation and Dissipation Times}

The preceding sections demonstrate that the FPD produces a measurable response in the presence of fog and that the response is correlated with $b_{\text {ext }}$ in the absence of external interferences. An additional assessment focused on how well the output of the FPD could be used to determine when fog was present and when it was not. For routine fog collection at the test site, fog has been assumed to be present when visibility drops below $0.5 \mathrm{~km}\left(b_{\text {ext }}>6 \mathrm{~km}^{-1}\right)$ and remains below that threshold for 5 minutes. To reduce fog

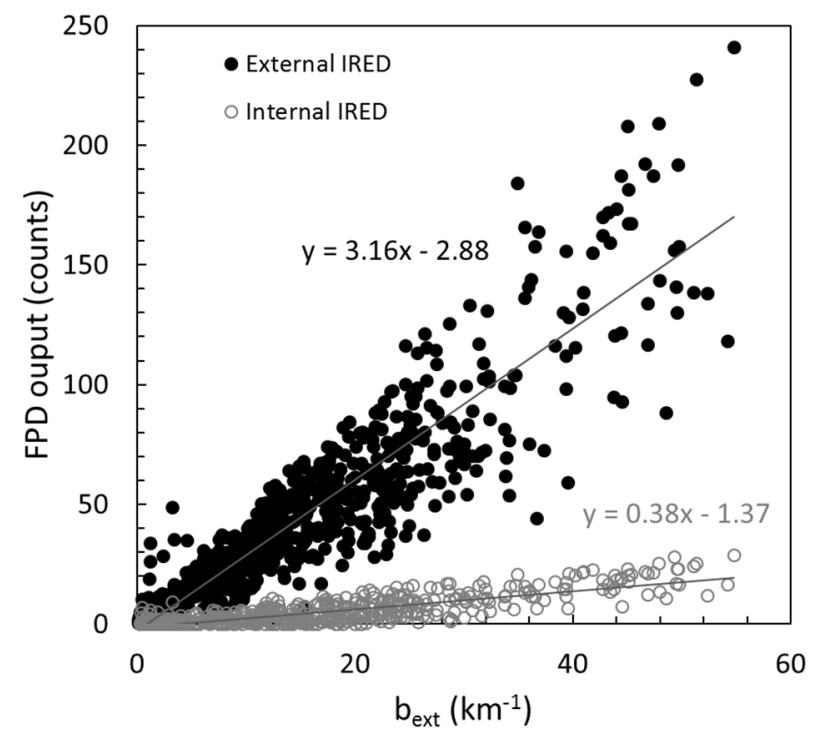

Fig. 4. FPD output vs. $b_{\text {ext }}$ values derived from a collocated visibility monitor for 15 fog events. 5-minute-averaged data is shown for two sensors, one configured with an internal IRED (open circles) and one configured with an external IRED (filled circles). misidentification, a second condition requires relative humidity to be greater than $85 \%$. Historically, these conditions have resulted in good fog identification with few false positives. For comparison purposes, similar conditional requirements were used for the FPD. Threshold values in the case of the FPD were 5 counts for the internal IRED sensors and 10 counts for the external IRED sensors. These thresholds were found to exclude the majority of background noise while capturing changes in output resulting from the presence of fog. Fog was assumed to be present until visibility or FPD output fell below threshold values for 2 minutes. Many of the fog events encountered during this study were intermittent, with foggy conditions interspersed with one or more clear periods.

Based on these conditions, fog formation and dissipation times were acquired from the visibility monitor and from each of the FPD sensors. The fog status (present or absent) was then assigned in 1 minute increments for each FPD sensor and for the visibility monitor over the duration of the study period. For each fog event, a determination was made of the percentage of time in which each individual FPD sensor was in agreement with the visibility monitor, with both simultaneously indicating the presence or absence of fog. Those results are compiled in Fig. 5, which shows the range of agreement between the visibility monitor and each FPD sensor for the 15 fog events in which the visibility monitor was unobstructed. Good agreement was observed

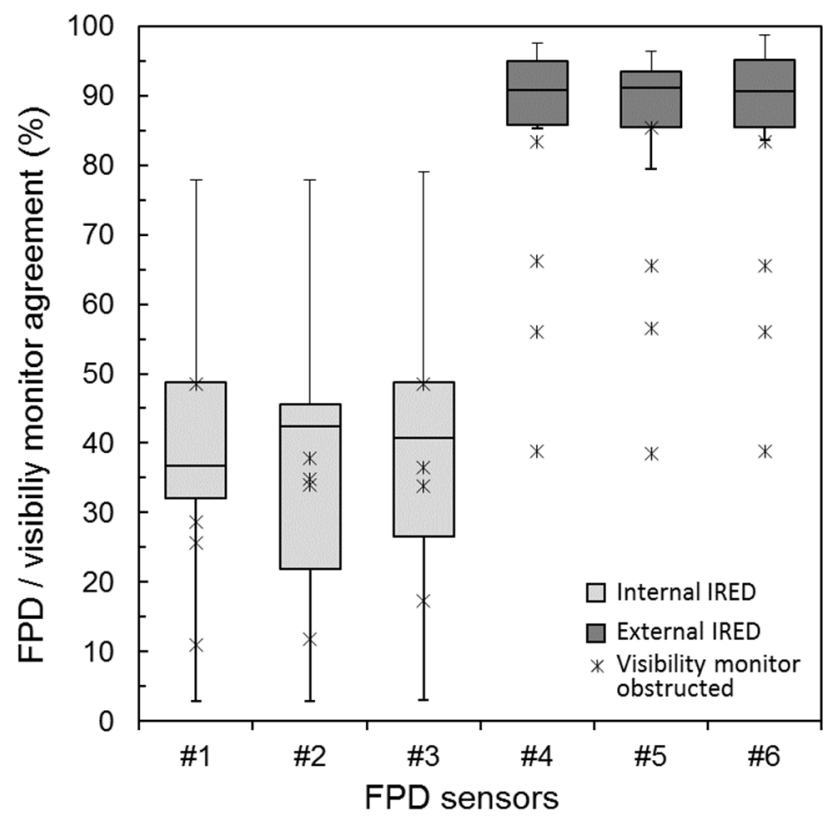

Fig. 5. Box plot showing the percentage of time that individual FPD sensors (\#1-6) and the visibility sensor simultaneously indicated that fog was present during each of 15 fog events. The central solid line represents the median value, the box represents the $25^{\text {th }}$ and $75^{\text {th }}$ percentiles, and the error bars represent the $10^{\text {th }}$ and $90^{\text {th }}$ percentiles. Agreement is shown for each of the internal IRED sensors (light gray) and external IRED sensors (dark gray). The individual data points represent the four fog events with observed external interferences that affected the visibility monitor data. 
for the sensors that utilized an external IRED. The median level of agreement was $91 \%$, with most of the discrepancy occurring during periods of optically thin fog when signals were marginally above or below threshold values for both instruments. Agreement was much worse for the sensors which used internal IREDs, with median values ranging between $36 \%$ and $42 \%$. This was due primarily to the sensors' loss of backscatter signal after sunrise. One event that occurred entirely after sunrise was not detected by any of the internal IRED sensors, resulting in $0 \%$ agreement with the visibility monitor. For completeness, visibility monitor and FPD sensor agreement for the four events in which the visibility monitor was affected by spider webs is also shown in Fig. 5 as individual data points.

\section{Performance in Clear Conditions}

In fog-free conditions, $3 \sigma$ noise in the FPD measurements was approximately 6.5 counts for sensors using the external IRED. Based on the correlation in Fig. 4, this corresponds to an extinction coefficient of approximately $3 \mathrm{~km}^{-1}$, or a visibility of $1 \mathrm{~km}$. Therefore, at visibilities greater than $1 \mathrm{~km}$, the FPD is unable to discern changes in visibility. If present at concentrations high enough to reduce visibility below $1 \mathrm{~km}$, haze or dust would be detected by the FPD and would not be distinguishable from fog. However, a relative humidity threshold could be used to discriminate between fog and dusty or smoky conditions.

Occasionally the FPD and visibility monitor exceeded the minimum threshold values that indicated the presence of fog when no fog was actually present, most often as a result of insects or spider webs in or near the optical sensing volumes. Spider webs were particularly disruptive due to their ability to elevate backscatter signals for long periods of time if not removed promptly. During non-foggy conditions, the FPD tended to be more prone to these interferences than the visibility monitor. The visibility monitor indicated the presence of fog during non-foggy conditions twice during the study, once due to heavy rain and once due to observed spider webs.

The minimum RH and 5 minute delay conditions helped eliminate some, but not all, false indications of fog from the FPD during clear conditions. The duration of time that each sensor recorded fog presence during non-foggy periods is shown in Fig. 6. Also included in Fig. 6 is the number of times in which false positives occurred for each sensor, and for reference, the cumulative duration of fog identified during fog events for each sensor. Misidentification of fog during non-foggy periods occurred on more occasions and for longer durations for the FPD sensors that relied on the internal IRED. The majority of the misidentification occurred during a 10 day period early in the study when spider webs were repeatedly observed over the internal IRED sensors which were all located on one side of the FPD enclosure. It was not clear why that side of the enclosure attracted more webs than the other, or alternatively, why the internal IRED sensor configuration attracted more webs than the external IRED configuration.

\section{Relationship with $L W C$}

LWC can play an important role in fog chemistry and

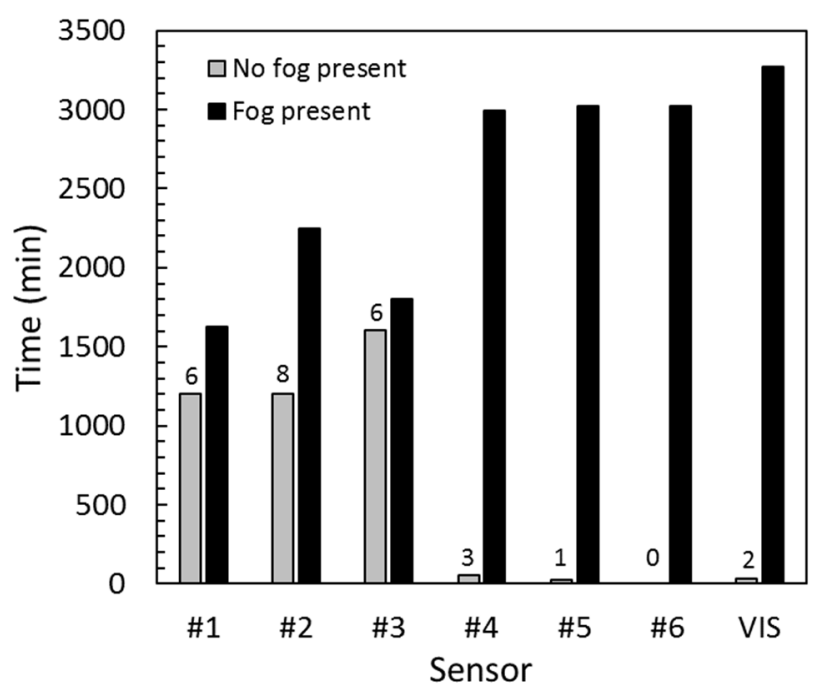

Fig. 6. Cumulative time that the FPD sensors (\#1-6) and the visibility monitor (VIS) satisfied the conditions that indicated the presence of fog. The black columns represent the total time that fog was identified during the 19 fog events. The gray columns represent the total time that fog was misidentified during non-foggy periods. The number of individual episodes in which fog was misidentified during non-foggy periods is also indicated.

water deposition flux. Therefore, the potential for the FPD to provide useful estimates of LWC was of interest. With no direct LWC measurements made during this study, fog LWC was determined based on the mass of fog water collected by the CHRCC during each fog event. Comparisons between LWC and FPD output were therefore made on an eventaveraged basis. No sample was collected during the shortest fog event, leaving 18 fog events for LWC analysis. Fog LWC was calculated following the approach of Demoz et al. (1996), which considers the rate of air flowing through the CHRCC, the rate of fog water collection, the drop-sizedependent collection efficiency of the CHRCC, and an assumed fog droplet distribution. After determining LWC, it was compared directly to the average output of an external IRED sensor for each event (Fig. 7(a)). LWC generally increased as the average response of the FPD increased, though variability was more pronounced at higher LWC values. A logarithmic regression of the data produces a root mean squared error (RMSE) of $0.03 \mathrm{~g} \mathrm{~m}^{-3}$ and a mean absolute percentage error (MAPE) of $22 \%$. This suggests that the FPD output can provide an indication of fog LWC, at least on an event-averaged basis. However, considering the relatively small sample size and limited LWC range encountered during this study, this should be considered a preliminary conclusion.

A number of researchers have used power law functions to characterize the relationship between visibility and LWC (Gultepe et al., 2006; Yamaguchi et al., 2015) or between $b_{\text {ext }}$ and LWC (Tomasi and Tampieri, 1976; Kunkle et al., 1984). These relationships take the form visibility $=a(L W C)^{b}$ or $\mathrm{b}_{\mathrm{ext}}=\mathrm{a}(\mathrm{LWC})^{\mathrm{b}}$, where the terms $a$ and $b$ are empirically derived. The two forms of the equation can be interconverted 

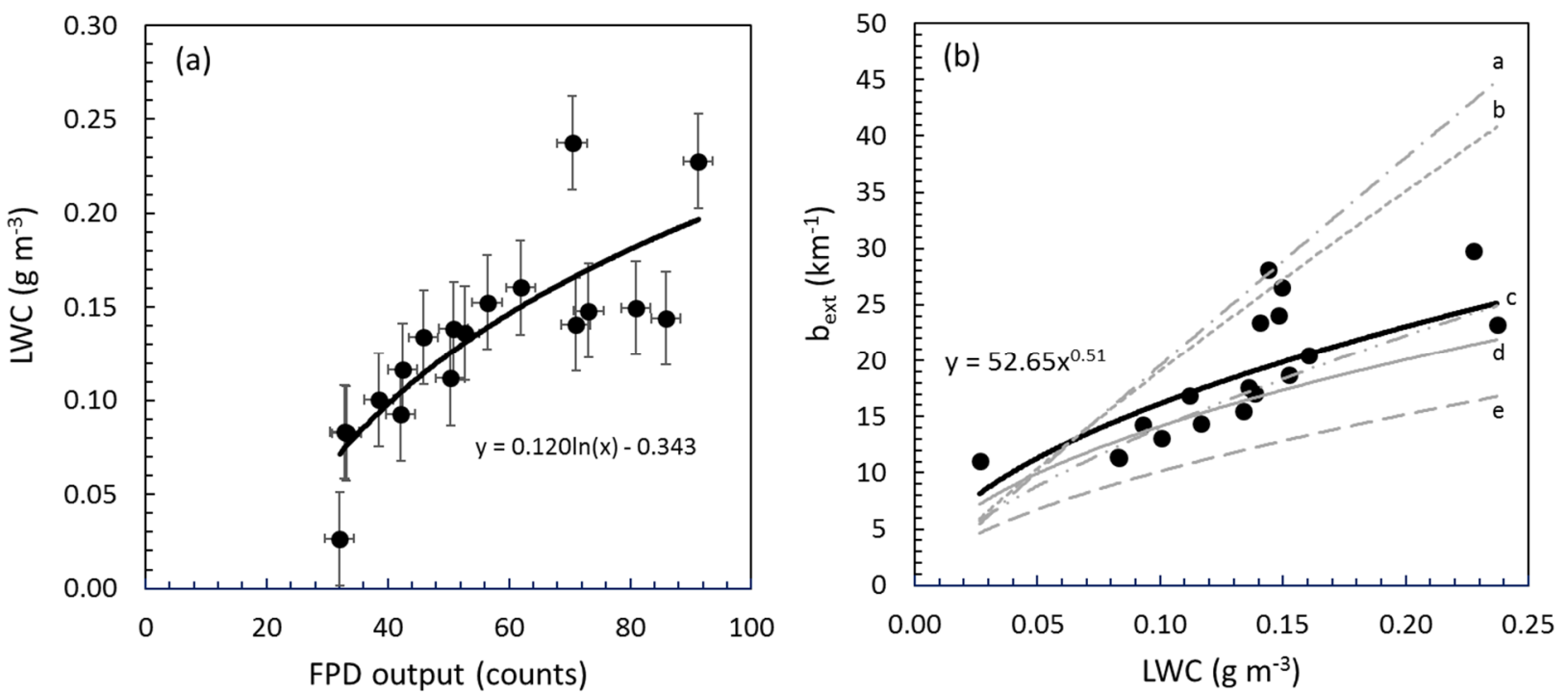

Fig. 7. (a) Calculated LWC as a function of FPD output for a sensor configured with an external IRED for 18 fog events. Error bars for LWC represent RMSE based on a comparison between CHRCC and Gerber PVM-100 data in Demoz et al. (1996). Error bars for FPD output represent the RMSE for fog-event-averaged output for the three sensors with external IREDs. (b) Extinction coefficient ( $b_{\text {ext }}$ ) vs. LWC (filled circles) and power law regression (black line) for the current study. Included for comparison are power law regressions from (a) Gultepe et al. (2006), (b) Kunkel et al. (1984), (c) Tomasi and Tampieri (1976), and (d) and (e) Yamaguchi et al. (2015).

using Eq. (3) with an appropriate contrast ratio. To compare data from the FPD to previous findings, the average output from an FPD sensor with external IRED for each fog event was converted to $b_{\text {ext }}$ using the linear regression presented in Fig. 4 and then graphed as a function of LWC (Fig. 7(b)). Also included in Fig. 7(b) is the power law regression for the current study, which yielded an RMSE $=0.06 \mathrm{~g} \mathrm{~m}^{-3}$ and $\mathrm{MAPE}=36 \%$. Power law regressions established by previous studies indicate similar trends and generally bound the data obtained from the FPD. Gultepe et al. (2006) shows that error in the relationship between LWC and $b_{\text {ext }}$ or visibility can be reduced by including droplet number concentration, which can vary significantly for a given LWC and therefore impact visibility.

\section{CONCLUSIONS}

A small, low-cost, commercially available proximity sensor, the VCNL4200, has been adapted for use as a fog presence detector, providing an inexpensive alternative to traditional devices used for fog detection. Its low cost makes it an economically feasible solution for low-budget fog studies, high-spatial-density fog monitoring networks, and applications such as the evaluation of fog harvesting sites. The sensor's small size and low power consumption also allow it to be mounted on tall towers or even drones, thereby aiding studies of the vertical extent of fog or its optical density variation with height.

The output values of the individual VCNL4200 sensors were found to be strongly correlated with one another, indicating that these sensors can be used interchangeably for fog detection without calibration. Field evaluations demonstrated that the new FPD is capable of distinguishing between foggy and clear conditions. The times of fog formation and dissipation determined by the FPD were in good agreement with those determined by a collocated visibility monitor, with two qualifications. First, the FPD sensors that utilized the VCNL4200's internal IRED performed poorly after sunrise due to overcompensation by the sensor's background light cancellation feature. The inclusion of a more powerful, external IRED was required for fog detection during the daytime. Second, interfering objects in the sensing volume of either the FPD or the visibility monitor produced misleading results. Insects and spider webs were the primary concern, resulting in false positive fog identification during intermittent fog events or even completely clear conditions. Implementing a minimum relative humidity condition and a delay after the first detection of elevated signals helped eliminate false positive events. To further distinguish false positives from actual fog events recorded by the FPD, the use of two or more FPD sensors at a given location is recommended. The determination of fog presence can then be based on agreement between two sensors or between two of three sensors. Over the course of this study, interference rarely affected more than one sensor of each configuration at any given time. This suggests that the concurrent use of multiple sensors-a practical option due to the low incremental cost of additional sensors - would improve the reliability of fog detection.

\section{ACKNOWLEDGMENTS}

The author gratefully acknowledges the financial support of Susquehanna University and the Department of Earth and Environmental Sciences. 


\section{REFERENCES}

Baumgardner, R.E., Kronmiller, K.G., Anderson, J.B., Bowser, J.J. and Edgerton, E.S. (1997). Development of an automated cloud water collection system for use in atmospheric monitoring networks. Atmos. Environ. 31: 2003-2010.

Boris, A.J., Lee, T., Park, T., Choi, J., Seo, S.J. and Collett Jr, J.L. (2016). Fog composition at Baengnyeong Island in the eastern Yellow Sea: Detecting markers of aqueous atmospheric oxidations. Atmos. Chem. Phys. 16: 437453.

Burkard, R., Bützberger, P. and Eugster, W. (2003). Vertical fogwater flux measurements above an elevated forest canopy at the Lägeren research site, Switzerland. Atmos. Environ. 37: 2979-2990.

Carrillo, J.H., Emert, S.E., Sherman, D.E., Herckes, P. and Collett Jr, J.L. (2008). An economical optical cloud/fog detector. Atmos. Res. 87: 259-267.

Decesari, S., Sowlat, M.H., Hasheminassab, S., Sandrini, S., Gilardoni, S., Facchini, M.C., Fuzzi, S. and Sioutas, C. (2017). Enhanced toxicity of aerosol in fog conditions in the Po Valley, Italy. Atmos. Chem. Phys. 17: 7721-7731.

Demoz, B.B., Collett Jr, J.L. and Daube Jr, B.C. (1996). On the Caltech active strand cloud water collectors. Atmos. Res. 41: 47-62.

Ervens, B., Wang, Y., Eagar, J., Leaitch, W.R., Macdonald, A.M., Valsaraj, K.T. and Herckes, P. (2013). Dissolved organic carbon (DOC) and select aldehydes in cloud and fog water: The role of the aqueous phase in impacting trace gas budgets. Atmos. Chem. Phys. 13: 5117-5135.

Facchini, M.C., Fuzzi, S., Zappoli, S., Andracchio, A., Gelencsér, A., Kiss, G., Krivácsy, Z., Mészáros, E., Hansson, H.C., Alsberg, Z. and Zebühr, Y. (1999). Partitioning of the organic aerosol component between fog droplets and interstitial air. J. Geophys. Res. 104: 26821-26832.

Forthun, G.M., Johnson, M.B., Schmitz, W.G., Blume, J. and Caldwell, R.J. (2006). Trends in fog frequency and duration in the southeast United States. Phys. Geogr. 27: 206-222.

Friedlein, M.T. (2004). Dense fog climatology: Chicago O’Hare International Airport July 1996 - April 2002. Bull. Am. Meteorol. Soc. 85: 515-517.

Gelencser, A., Sallai, M., Krivacsy, Z., Kiss, G. and Meszaros, E. (2000). Voltammetric evidence for the presence of humic-like substances in fog water. Atmos. Res. 54: 157-165.

Giulianelli, L., Gilardoni, S., Tarozzi, L., Rinaldi, M., Decesari, S., Carbone, C., Facchini, M.C. and Fuzzi, S. (2014). Fog occurrence and chemical composition in the Po valley over the last twenty years. Atmos. Environ. 98: 394-401.

Gultepe, I., Müller, M.D. and Boybeyi, Z. (2006). A new visibility parameterization for warm-fog applications in numerical weather prediction models. J. Appl. Meteor. Climatol. 45: 1469-1480.

Gultepe, I., Tardif, R., Michaelides, S.C., Cermak, J., Bott, A., Bendix, J., Muller, M.D., Pagowski, M., Hansen, B.,
Ellrod, G., Jacobs, W., Toth, G. and Cober, S.G. (2007). Fog research: A review of past achievements and future perspectives. Pure Appl. Geophys. 164: 1121-1159.

Herckes, P., Chang, H., Lee, T. and Collett, J.L. (2007). Air pollution processing by radiation fogs. Water Air Soil Pollut. 181: 65-75.

Kunkel, B.A. (1984). Parameterization of droplet terminal velocity and extinction coefficient in fog models. $J$. Climate Appl. Meteor. 23: 34-41.

Meyer, M.B. and Lala, G.G. (1990). Climatological aspects of radiation fog occurrence at Albany, New York. $J$. Clim. 3: 577-586.

Michna, P., Schenk, J., Werner, R.A. and Eugster, W. (2013). MiniCASCC - A battery driven fog collector for ecosystem research. Atmos. Res. 128: 24-34.

Michna, P., Werner, R.A. and Eugster, W. (2015). Does fog chemistry in Switzerland change with altitude? Atmos. Res. 151: 31-44.

National Oceanic and Atmospheric Administration (NOAA) (2017). Surface weather observations and reports, Federal Meteorological Handbook No. 1. Washington, DC.

Roach, W.T., Brown, R., Caughey, S.J., Garland, J.A. and Readings, C.J. (1976). The physics of radiation fog: I-a field study. Q. J. R. Meteorolog. Soc. 102: 313-333.

Simon, S., Klemm, O., El-Madany, T., Walk, J., Amelung, K., Lin, P.H., Chang, S.C., Lin, N.H., Engling, G., Hsu, S.C., Wey, T.H., Wang, Y.N. and Lee, Y.C. (2016). Chemical composition of fog water at four sites in Taiwan. Aerosol Air Qual. Res. 16: 618-631.

Straub, D.J., Hutchings, J.W. and Herckes, P. (2012). Measurements of fog composition at a rural site. Atmos. Environ. 47: 195-205.

Straub, D.J. (2017). Radiation fog chemical composition and its temporal trend over an eight year period. Atmos. Environ. 148: 49-61.

Tardif, R. and Rasmussen, R.M. (2007). Event-based climatology and typology of fog in the New York City region. J. Appl. Meteor. Climatol. 46: 1141-1168.

Tav, J., Masson, O., Burnet, F., Paulat, P., Bourrianne, T., Conil, S. and Pourcelot, L. (2018). Determination of fogdroplet deposition velocity from a simple weighing method. Aerosol Air Qual. Res. 18: 103-113.

Thalmann, E., Burkard, R., Wrzesinsky, T., Eugster, W. and Klemm, O. (2002). Ion fluxes from fog and rain to an agricultural and a forest ecosystem in Europe. Atmos. Res. 64: 147-158.

Tomasi, C. and Tampieri, F. (1976). Features of the proportionality coefficient in the relationship between visibility and liquid water content in haze and fog. Atmos. 14: 61-76.

van Oldenborgh, G.J., Yiou, P. and Vautard, R. (2010). On the roles of circulation and aerosols in the decline of mist and dense fog in Europe over the last 30 years. Atmos. Chem. Phys. 10: 4597-4609.

Van Schalkwyk, L. and Dyson, L.L. (2013). Climatological characteristics of fog at Cape Town International airport. Weather Forecasting 28: 631-646.

Vishay Intertechnology, Inc. (2019). High sensitivity long distance proximity and ambient light sensor with I2C 
interface. VCNL4200 datasheet, Document Number: 84430, Rev 1.4.

Wang, Y., Zhang, J., Marcotte, A.R., Karl, M., Dye, C. and Herckes, P. (2015). Fog chemistry at three sites in Norway. Atmos. Res. 151: 72-81.

Weiss-Penzias, P., Fernandez, D., Moranville, R. and Saltikov, C. (2018). A low cost system for detecting fog events and triggering an active fog water collector. Aerosol Air Qual. Res. 18: 214-223.

World Meteorological Association (WMO) (2018). Technical Regulations, Basic Documents No. 2, Volume II Meteorological Service for International Air Navigation, Geneva, Switzerland.
Yamaguchi, T., Katata, G., Noguchi, I., Sakai, S., Watanabe, Y., Uematsu, M. and Furutani, H. (2015). Long-term observation of fog chemistry and estimation of fog water and nitrogen input via fog water deposition at a mountainous site in Hokkaido, Japan. Atmos. Res. 151: 82-92.

Received for review, November 26, 2019

Revised, February 28, 2020 Accepted, March 12, 2020 